\title{
The Favored Mechanism for Coping with Acute Cold Stress: Upregulation of miR-210 in Rats
}

\author{
Wenjin Guo ${ }^{a}$ Shuai Lian ${ }^{a} \quad$ Li Zhen $^{\mathrm{a}}$ Shucheng Zang ${ }^{\mathrm{a}}$ Yan Chen ${ }^{\mathrm{a}}$ Limin Lang ${ }^{\mathrm{a}}$ \\ Bin $\mathrm{Xu}^{\mathrm{a}}$ Jingru Guo ${ }^{\mathrm{a}}$ Hong Jia Jianfa Wang ${ }^{\mathrm{a}}$ Shoupeng Fu ${ }^{\mathrm{c}}$ Liping Zhang ${ }^{\mathrm{b}}$ \\ Huanmin Yang ${ }^{a}$ \\ ${ }^{a}$ College of Animal Science and Veterinary Medicine, Heilongjiang Bayi Agricultural University, Daqing, \\ ${ }^{b}$ College of Food Science, Heilongjiang Bayi Agricultural University, Daqing, 'College of Veterinary \\ Medicine, Jilin University, Changchun, China
}

\section{Key Words}

Cold stress $\cdot \operatorname{miR}-210 \cdot$ Proliferation • Cell death • Glycolysis • Mitochondrial respiration

\begin{abstract}
Background/Aims: The main aim of this study was to determine the mechanisms by which rno-miR-210-3p affects changes in gene expression, metabolism, apoptosis and proliferation of cells under acute cold stress (ACS) conditions. Methods: The treatment group ( $n=6$, weight $340 \pm 20 \mathrm{~g}$ ) was exposed to ACS (temperature $4 \pm 0.5^{\circ} \mathrm{C}$, relative humidity $45 \pm 0.5 \%$ ) and the control group ( $n=6$, weight $340 \pm 20 \mathrm{~g}$ ) to normal temperature (NT) (temperature $24 \pm 0.5^{\circ} \mathrm{C}$, relative humidity $45 \pm 0.5 \%$ ). Rat liver samples were collected for qRT-PCR and western blot analyses to detect relative expression of rno-miR-210-3p, ISCU, Rap1b, ATP1b1, GPD1, E2F3, RAD52, PSMB6 and GPD2. For cell experiments, 100 pmol/dish rno-miR-210-3p mimic and 150 $\mathrm{pmol} / \mathrm{dish}$ rno-miR-210-3p inhibitor were used. Mitochondrial glucose flux and glycolysis were measured using the XFe24 Extracellular Flux Analyzer. Cells were collected for apoptosis analysis $24 \mathrm{~h}$ after transfection and proliferation was quantified using the WST-1 Cell Proliferation and Cytotoxicity Assay Kit (Beyotime, Shanghai, China), according to the manufacturer's instructions. Results: In the rat experiment, expression of rno-miR-210-3p under ACS was increased sharply while ISCU, E2F3, RAD52, and PSMB6 levels declined, along with protein expression of ISCU and PSMB6. In cell experiments, ISCU, Rap1b, ATP1b1, GPD1, E2F3, RAD52, PSMB6 and GPD2 genes were downregulated while ISCU and PSMB6 protein expression decreased with upregulation of rno-miR-210-3p. Conversely, in response to decreased rnomiR-210-3p expression, ISCU, E2F3, RAD52, PSMB6 and GPD2 genes were upregulated, in addition to ISCU and PSMB6 proteins. Upregulation of miR-210 inhibited cell proliferation and induced cell death whereas its downregulation promoted cell proliferation. Upregulation or downregulation of miR-210 promoted glycolysis and mitochondrial respiration of BRL cells.
\end{abstract}

Huanmin Yang

and Liping Zhang

KARGER
College of Animal Science and Veterinary Medicine. Heilongjiang Bayi Agricultura University, NO.5 Xinfeng Road, High and New Technology Industrial Development

Zone, Daqing 163000 (China); Tel. +86-0459- 6819201,

E-Mail yanghuanmin@aliyun.com; ZLP77@126.com 
However, downregulation of miR-210 caused acid production in cells. Conclusion: Expression of rno-miR-210-3p is significantly increased under ACS. Upregulation of rno-miR-210-3p inhibits the expression of ISCU, Rap1b, ATP1b1, GPD1, E2F3, RAD52, PSMB6 and GPD2 genes, promotes glycolysis of liver and enhances the mitochondrial respiratory capacity of cells, but may also cause cell death. Our findings collectively indicate that regulation of rno-miR-210-3p is a preferential mechanism of choice used by the body to cope with ACS.

(C) 2018 The Author(s)

Published by S. Karger AG, Basel

\section{Introduction}

In high latitude areas and cold winter conditions, humans and animals experience stress reactions in response to sudden cold air outbreaks. Long - or short-term cold exposure may lead to growth retardation [1], loss of genetic information [2] and immune suppression [3]. In general, cold stress is classified into acute cold stress (ACS) and chronic cold stress according to exposure time to low environmental temperature [4]. The cold exposure time of ACS is in the range of tens of minutes to 1 day [5]. The liver may generate a series of anti-cold stress reactions involving alterations in expression patterns of specific genes and proteins to enhance protection and alleviate the adverse effects of ACS [6]. miRNAs, a class of endogenous small non-coding RNA molecules composed of $\sim 22$ nucleotides [7], play an extremely important role in this regulatory process [8]. These molecules usually target multiple mRNAs and modulate gene expression by inhibiting translation or degrading target mRNAs [9]. Over $60 \%$ of all mammalian mRNAs are predicted targets of miRNAs [10] and miRNAs constitute a sizeable class of regulators ( $\geq 950$ different miRNAs in humans according to miRBase release 15 in April 2010; http://www.mirbase.org/), even outnumbering kinases and phosphatases, indicating pervasive roles in regulation of cellular processes.

Several studies to date have focused on cardiac disease and tumors in relation to miR-210 [11, 12]. Moreover, miR-210 is strongly linked with the hypoxia pathway [13] and upregulated in response to hypoxia-inducible factors [14]. Recent research suggests that miR-210 additionally plays important roles in regulation of energy metabolism [15], immunity [16], cell proliferation and apoptosis [17]. The miRNA has been shown to regulate transcription factors, proteasome components [18], iron sulfur synthase [19], DNA repair processes and glycerol-3-phosphate dehydrogenase 2. In 2017, Zhen and co-workers constructed two small RNA libraries with sera of rats either subjected to cold $\left(4^{\circ} \mathrm{C} \pm 0.5^{\circ} \mathrm{C}\right.$ for $12 \mathrm{~h}$ ) or normal temperature $\left(24^{\circ} \mathrm{C} \pm 0.5^{\circ} \mathrm{C}\right.$ for $\left.12 \mathrm{~h}\right)$ conditions via deep sequencing, which led to the discovery that miR-210 is significantly linked with cold stress [20]. In view of its multiple regulatory functions, miR-210 is proposed to play a critical role in ACS. Accordingly, in the current study, we focused on the effects of miR-210 on rat liver under ACS and the underlying mechanisms.

\section{Materials and Methods}

\section{Animals and tissues}

Adult male Wister rats were purchased from Beijing Vital River Laboratory Animal Technology Co., Ltd. (Beijing, China). Rats were housed in the animal center of Heilongjiang Bayi Agriculture University and received humane care according to the criteria outlined in the guide for the care and Use of Laboratory Animals (National Institutes of Health publication 86-23, 1985 revision). All experimental protocols involving animals were approved by the Animal Care and Use Committee of Heilongjiang Bayi Agricultural University (Daqing, China). Animals had free access to standard chow and water. Rats were habituated to an artificial intelligence climate chamber for 2 weeks with pre-feeding before the initiation of the experiment (controlled constant temperature (Tm) of $24 \pm 0.5^{\circ} \mathrm{C}$ and Relative humidity (Rh) $45 \pm 0.5 \%, 12 \mathrm{~h} / 12 \mathrm{~h}$ light/ dark cycle, with free access to food and water). After the pre-feeding period, rats were randomly divided into two groups (control and treatment). Each group contained six rats that were equally randomly assigned to two artificial intelligence climate chambers. The treatment group (six adult male Wistar rats, $340 \pm 20 \mathrm{~g}$ ) 
was exposed to ACS (Tm $4 \pm 0.5^{\circ} \mathrm{C}$, Rh $45 \pm 0.5 \%$ ) for $12 \mathrm{~h}$ during the dark cycle and the control group (six adult male Wistar rats, $340 \pm 20 \mathrm{~g}$ ) to normal temperature (NT) conditions ( $\mathrm{Tm} 24 \pm 0.5^{\circ} \mathrm{C}$, Rh $45 \pm 0.5 \%$ ) for $12 \mathrm{~h}$ during the dark cycle. Subsequently, all animals were injected with an anasthetic, pentobarbital sodium $(1.5 \%, 0.3 \mathrm{~mL} / 100 \mathrm{~g})$, for collection of livers.

\section{Cell culture and reagents}

BRL cells (normal rat hepatocytes) were obtained from the American Type Culture Collection (Rockville, MD, USA), and maintained in the recommended culture conditions. All cell lines were maintained in DMEM supplemented with $10 \%$ fetal bovine serum (Gibco, Carlsbad, CA, USA) at $37^{\circ} \mathrm{C}$ in a $5 \% \mathrm{CO}_{2}$ humidified incubator. Cells were grown in a monolayer and routinely passaged two or three times a week. Dimethyl sulfoxide (DMSO) was purchased from Sigma (St. Louis, MO, USA)

\section{Cell transfection}

The rno-miR-210-3p mimic (catalog \#M518200-9000) and rno-miR-210-3p inhibitor (catalog \# M518200-9000) were purchased from Sangon Biotech (Shanghai, China). The sequences were as follows: rno-miR-210-3p mimic, sense 5'-CUGUGCGUGUGACAGCGGCUGA3' and antisense 5'-AGCCGCUGUCACACGCACAGUU-3'; Mimic Negative Control (MNC), sense 5'-UUCUCCGAA CGUGUCACGUTT-3' and antisense 5'-ACGUGACACGUUCGGAGAATT-3'; rno-miR210-3p inhibitor, sense 5'-UCAGCCGCUGUCACACGCACAG-3'; Inhibitor Negative Control (INC), sense 5'-CAGUACUUUUGUGUAGUACAA-3'. Cells were cultured in a $60 \mathrm{~mm} \times 15 \mathrm{~mm}$ cell culture dish (Life Science, Oneonta, USA) and divided into four groups: MNC, INC, rno-miR-210-3p mimic (210M), rno-miR-210-3p inhibitor (210I). At the time of transfection, cells that reached 50\%-60\% confluence were transfected using the Lipofectamine RNAiMAX Reagent (catalog \#13778150, Invitrogen, Carlsbad, CA, USA) system according to the manufacturer's instructions. We used concentrations of $100 \mathrm{pmol} / \mathrm{dish}$ rno-miR-210-3p mimic and $150 \mathrm{pmol} / \mathrm{dish}$ rno-miR-210-3p inhibitor. Cells were collected for analysis at $24 \mathrm{~h}$ after transfection.

\section{Real-time PCR}

Total RNA was extracted from BRL cell lines and rat livers using TRIzol reagent (Life Technologies, Carlsbad, CA, USA). Following DNasel treatment, $2 \mu \mathrm{g}$ total RNA was reverse-transcribed (RT) using the PrimeScript $^{\mathrm{TM}}$ RT reagent Kit (TaKaRa, Tokyo, Japan) to synthesize cDNA sequences. RT-PCR analysis of gene expression was performed using $2 \mu \mathrm{L}$ cDNA and SYBR ${ }^{\circledR}$ Green Premix Ex Taq ${ }^{\mathrm{TM}}$ II (TaKaRa), as recommended by the manufacturer, on the CFX96 system (Bio-Rad, California, USA). miRNA was transcribed into cDNA using a specific oligo-dT (RT primer). Next, cDNA was combined with SYBR ${ }^{\circledR}$ Green Premix Ex $\mathrm{Taq}^{\mathrm{TM}} \mathrm{II}$, two miRNA primers (with sequences complementary to the miRNA) and $\mathrm{dH}_{2} \mathrm{O}$ to complete the RT-PCR reaction. miRNA expression was normalized using cel-miR-39-5p. The following primers were employed: cel-miR-39-5p: 5'-GTCGTATCCAGTGCAGGGTCCGAGGTATTCGCACTGGATACGACCAAGCT-3' (RT primer), 5'-GCGGCGGTCACCGGGTGTAAATC-3' (forward), 5'-ATCCAGTGCAGGGTCCGAGG-3' (reverse); rno-miR-210-3p:5'-GTCGTATCCAGTGCAGGGTCCGAGGTATTCGCACTGGATACGACTCAGCC-3' (RT primer), 5'-GCGGCGGCTGTGCGTGTGACAG-3' (forward), 5'-ATCCAGTGCAGGGTCCGAGG-3' (reverse). For mRNA detection, GAPDH (order No. B661204, Sangon Biotech, Shanghai, China) was used as an internal control to normalize gene expression values. The primer sequences used were as follows: 5'-AGGGGAAGATTGTGGATGC3' (forward), 5'-GGCGATGTCTGTGTTTTTGA-3' (reverse) for ISCU; 5'-CCCAGGCTCCCAAATCTT-3' (forward), 5'-CAAGGAAAGCAGAGGAAGGA-3' (reverse) for E2F3; 5'-TCCGTCACCCAGCAGAAT-3' (forward), 5'-TCCATAGCCCACATCCTCAT-3' (reverse) for RAD52; 5'-GACAAGGCTTTGCGTTGGTT-3' (forward), 5'-TTGGGACATCGTCAGTGTCT-3' (reverse) for Rap1b; 5'-GCCAATCGAGTGACTGACAA-3' (forward), 5'-GTGGAAACCAAGCTGGTACG-3' (reverse) for PSMB6; 5'-AAGGAGCAGCTCAAGGTTCA-3' (forward), 5'-AGCTCCTGATGTGTGCTGTG-3' (reverse) for GPD1; 5'-AGTAAGCCTGGCGTATGTGG-3' (forward), 5'-TCCAATGACGAGGATGTCAA-3' (reverse) for GPD2; 5'-GCGGATTCTATGGCTTTCCT-3' (forward), $5^{\prime}$-CGGATTTCAGTGTCCAAGGT-3' (reverse) for ATP1b1. Each PCR was run in triplicate at $95^{\circ} \mathrm{C}$ for $30 \mathrm{~s}$ followed by 40 cycles of $95^{\circ} \mathrm{C}$ for $5 \mathrm{~s}$ and $60^{\circ} \mathrm{C}$ for $30 \mathrm{~s}$, and a melt curve generated from $65^{\circ} \mathrm{C}$ to $95^{\circ} \mathrm{C}$ (increment $0.5^{\circ} \mathrm{C}, 5 \mathrm{~s}$ ). Comparative quantification was performed using the $2^{-\Delta \Delta \mathrm{Ct}}$ method. Each sample was analyzed in triplicate.

\section{KARGER}




\section{Cellular Physiology Cell Physiol Biochem 2018;46:2090-2102 \\ \begin{tabular}{l|l} 
and Biochemistry Published 10.1159/000489449 & $\begin{array}{l}\text { (c) } 2018 \text { The Author(s). Published by S. Karger AG, Basel } \\
\text { www.karger.com/cpb }\end{array}$
\end{tabular}}

Guo et al.: The Favored Mechanism for Coping With Acute Cold Stress: Upregulation of MiR-210 in Rats

Western blot analysis

Total proteins were isolated from cells and rat livers with RIPA lysis buffer (Beyotime, Shanghai, China) (50 mM Tris, pH 7.4; 150 mM NaCl; 1\% Triton X-100; 1\% sodium deoxycholate; 0.1\% SDS; sodium orthovanadate; sodium fluoride; EDTA, leupeptin; $1 \mathrm{mM}$ PMSF). Lysates and Tissue lysate were centrifuged at $12000 \mathrm{~g}$ for $5 \mathrm{~min}$ at $4^{\circ} \mathrm{C}$. Protein concentrations were determined with an Enhanced BCA Protein Assay Kit (Beyotime, Shanghai, China). Equal amounts $(25 \mu \mathrm{g})$ of cell/ hepatic tissue extracts were subjected to electrophoresis using $12 \%$ sodium dodecyl sulfate-polyacrylamide (SDS-PAGE) and subsequently transferred to PVDF membranes (Millipore, Darmstadt, Germany) for antibody blotting. Membranes were blocked and incubated with $\beta$-actin (1:15000), ISCU (1:800) or PSMB6 (1:500) antibody (Protein Tech Group, Chicago, IL, USA) overnight at $4^{\circ} \mathrm{C}$, followed by HRP-conjugated anti-mouse (1:1000) or anti-rabbit secondary antibody (1:1000) (Beyotime, Shanghai, China) at room temperature for $1 \mathrm{~h}$. Protein bands were visualized using a Beyo Enhanced Chemiluminescence reagent kit (Beyotime, Shanghai, China) according to the manufacturer's instructions.

\section{Glucose flux in mitochondria and glycolysis}

Glucose flux was measured using the XFe 24 Extracellular Flux Analyzer. During seahorse experiments, Seahorse Assay Medium (Sigma-Aldrich, D5030) supplemented with 1\% FBS was used with no glucose, pyruvate or glutamine included. Oxygen consumption rate (OCR) was measured using the XF Cell Mito Stress Test Kit (\#103015-100, Agilent Technologies, UK). Data were calculated from four independent measurements obtained at 5 min intervals at baseline and after addition of working concentrations of glucose $(10 \mathrm{mM})$, oligomycin $(5 \mu \mathrm{M})$, FCCP $(1 \mu \mathrm{M})$ and rotenone $(5 \mu \mathrm{M})$. Extracellular acidification rate (ECAR) was measured using the XF Glycolysis Stress Test Kit (\#103020-100, Aglilent Technologies, UK). Data were calculated from four independent measurements obtained at 5-min intervals at baseline and after the addition of the following compounds at working concentrations: glucose $(1 \mathrm{mM})$, amphotericin (1 $\mathrm{mM})$, and 2-DG (5 mM). The mean values from four replicate wells were recorded and presented as the mean \pm standard error of the mean (SEM). Experiments were repeated four times.

\section{Cell apoptosis and proliferation}

Apoptosis was evaluated via fluorescence-activated cell sorting after annexin V-FITC and propidium iodide staining. Adherent cells were detached with 2.5\% trypsin-EDTA and added to the culture supernatant. Cells were pelleted by centrifugation at $1000 \mathrm{rpm}$ for $5 \mathrm{~min}$, suspended in $300 \mu \mathrm{L} 1 \times$ binding buffer (FITC Annexin V Apoptosis Detection Kit I, BD Biosciences, USA) and incubated for 20 min with Annexin V-FITC (5 $\mu \mathrm{L})$ and propidium iodide $(5 \mu \mathrm{L})$. Flow cytometry was performed on a CytoFLEX flow cytometer, and data analyzed using CytExpert (Beckman Coulter, USA).

Cell proliferation was quantified using the WST-1 Cell Proliferation and Cytotoxicity Assay Kit (Beyotime, Shanghai, China) according to the manufacturer's instructions. Electronic coupling agent (1 mL) was added into WST-1, and $10 \mu \mathrm{L}$ WST-1 solution used for every $100 \mu \mathrm{L}$ culture medium.

\section{miRNA target prediction}

Analysis of predicted targets for rno-miR-210-3p was performed using the algorithms TargetScan (http://targetscan.org/), PicTar4 (http:// pictar.mdc-berlin.de/), miRDB (http://www.mirdb.org/miRDB/), and miRanda (http://www.microrna.org/ microrna).

\section{Statistical analysis.}

Images were produced using GraphPad prism software. Animals were randomly assigned to groups. In rat and cell studies, histological analysis was conducted in a blinded manner. Based on extensive experience with rat models of ACS and the planned analytical framework, we estimated the number of rats per group required to detect effects of interest at the $\mathrm{P}<0.05$ level of significance. The numbers of technical or biological replicates (independent experiments for cell culture or individual rats for in vivo experiments) in each group are specified in the respective figure legends. All data are presented as the mean \pm SEM or the mean \pm standard deviation (SD), as shown in the figure legends. Unpaired Student's t-tests, with no assumption of equal variance, were used for comparing the two groups. To determine goodness of fit correlation (R2), univariate linear regression was employed. ANOVA (general linear model) was applied for the comparison of more than two groups. In cases where overall F-tests were significant $(\mathrm{P}<0.05)$, post hoc comparisons using 
Tukey's method of adjustment were conducted to determine the location of significant pairwise differences. Analyses were performed using GraphPad prism 7.00 software (La Jolla, CA, USA).

\section{Results}

Bioinformatic analysis of miR-210

In total, 443 target genes of rno-miR-210-3p were predicted using Targetscan (101 target genes), miRanda (334 target genes), miRDB (33 target genes) and PicTar (15 target genes) (Fig. 1a). Eight genes from 30 candidates were screened for further analysis using the four software types (Fig. 1b). All predicted binding sites are shown in Fig. 1d. Gene ontology (GO) analysis revealed that rno-miR-210-3p is involved in five biological processes, including the BMP signaling pathway, negative regulation of activin receptor signaling, positive regulation of osteoblast differentiation, cellular response to lipopolysaccharides and cellular response to leukemia inhibitory factor. These biological processes

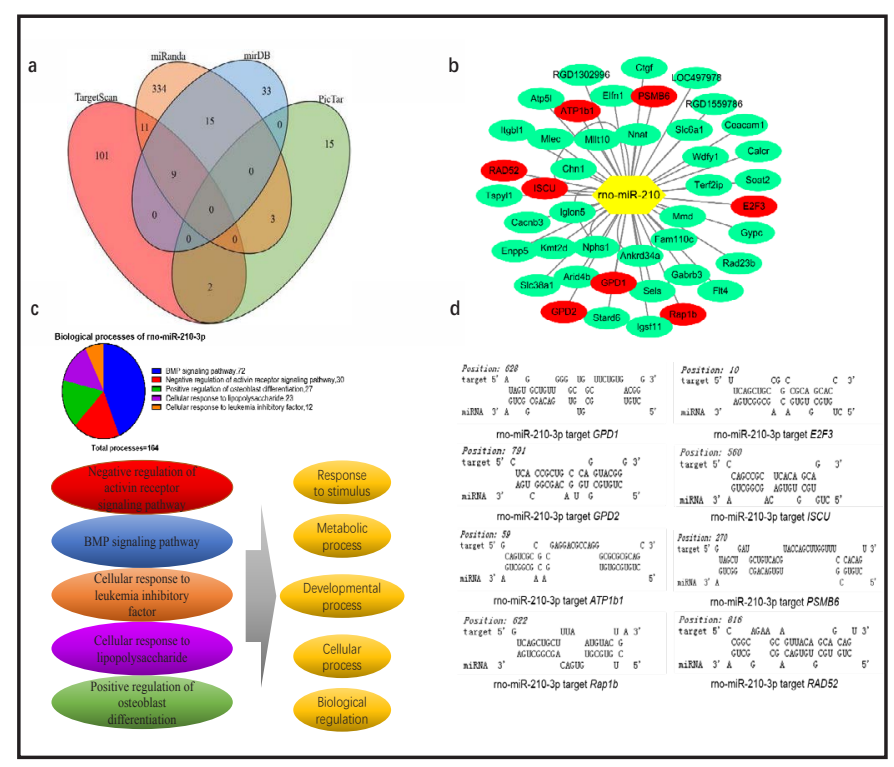

Fig. 1. Prediction of miR-210 target genes and biological functions. (a) Wayne map of target genes of rno-miR-210-3p. (b) Target genes of rno-miR-210-3p. The red sections represent target genes for subsequent validation. (c) Biological processes affected by rno-miR-210-3p. (d) Identification of potential mRNAs (GPD1, E2F3, GPD2, ISCU, ATP1b1, PSMB6, Rap1b and RAD52) that are direct targets of rno-miR-210-3p. All the target sites were predicted using RNAhybrid.

Fig. 2. Expression of rno-miR-210-3p normalized to cell-miR-39-5p. miRNA levels in BRL cells were detected via qRT-PCR. MNC, transfection with Mimic (duolex) Negative Control; $210 \mathrm{M}$, transfection with rno-miR210-3p mimic. INC, transfection with Inhibitor (single) Negative Control; 210I, transfection with rno-miR210-3p inhibitor. (a) Treatment with 60/100/150 pmol/dish rno-miR-210$3 p$ mimic or MNC for $12 / 24 h$. (b) Treatment with 60/100/150 pmol per dish rno-miR-210-3p inhibitor or INC

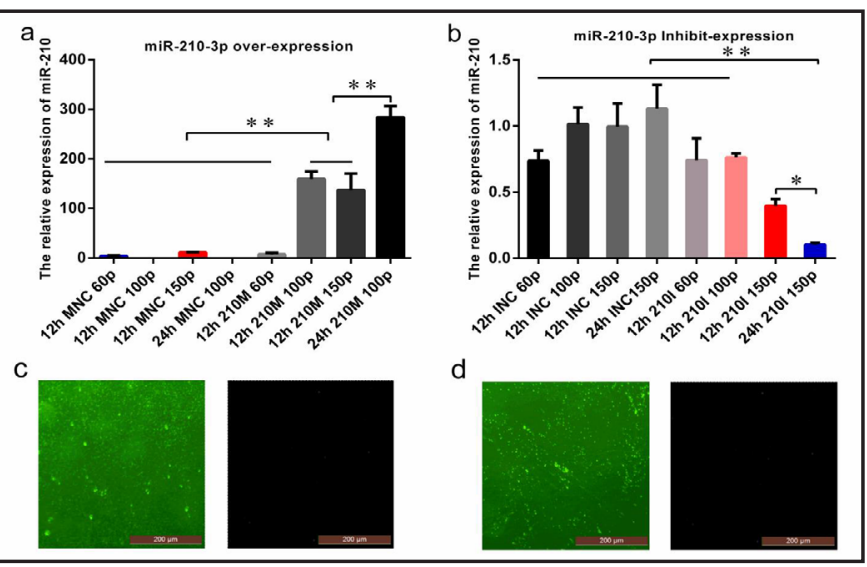
for $12 / 24 \mathrm{~h}$. (c) Green fluorescence with mimics was observed under an inverted fluorescence microscope after transfection for $24 \mathrm{~h}$ at 100 pmol/dish. (d) Green fluorescence with inhibitor was observed under an inverted fluorescence microscope after transfection for $24 \mathrm{~h}$ at $150 \mathrm{pmol} / \mathrm{dish}$. All data are presented as the mean \pm SEM. Data was analyzed using ANOVA. In cases where overall F-tests were significant $(P<0.05)$, post-hoc comparisons using Tukey's method of adjustment were conducted to determine the location of significant pairwise differences. ${ }^{* *} P<0.01 ;{ }^{*} P<0.05$.

\section{KARGER}




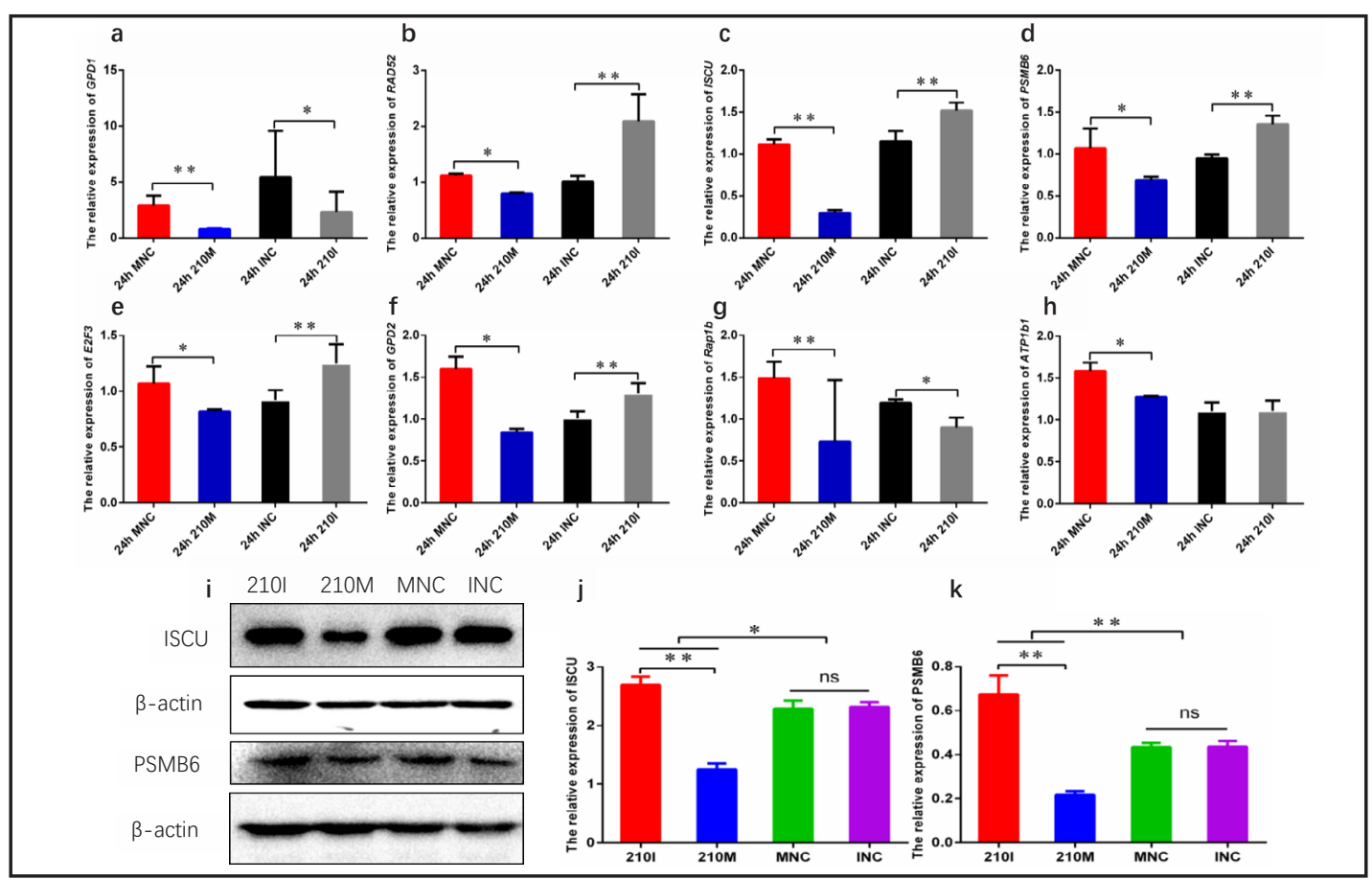

Fig. 3. Effects of rno-miR-210-3p on expression of ISCU, Rap1b, ATP1b1, GPD1, E2F3, RAD52, PSMB6 and GPD2 normalized to GAPDH. Effects of rno-miR-210-3p on protein expression of PSMB6 and ISCU. Relative protein expression was measured using ImageJ software (http://imagej.nih.gov/ij/) and normalized to $\beta$-actin. Protein expression in BRL cells was detected using western blot. MNC, Transfection with Mimic (duolex) Negative Control; 210M, Transfection with rno-miR-210-3p mimic. INC, Transfection with Inhibitor (single) Negative Control; 210I, Transfection with rno-miR-210-3p inhibitor. (a-h) qRT-PCR analysis of mRNA expression in BRL cells. a-h depict mean \pm SEM. in bar graphs $(n=3)$. All data were analyzed using t- test. ${ }^{* *} P<0.01{ }^{*} P<0.05$. (i) 210 I and INC cells were transfected with rno-miR-210-3p inhibitor and INC (single), respectively. 210M and MNC cells were transfected rno-miR-210-3p mimic and MNC (duplex), respectively. (j-k) Relative expression of ISCU and PSMB6. (i-k) Data were analyzed using ANOVA and presented as mean \pm SEM. $(n=3)$. In cases where overall F-tests were significant $(P<0.05)$, post hoc comparisons using Tukey's method of adjustment were conducted to determine the location of significant pairwise differences $\left({ }^{*} P<0.05,{ }^{* *} P<0.01\right)$.

eventually function in regulation, metabolic, developmental and cellular processes and response to stimuli (Fig. 1c).

\section{Transfection of mimics and inhibitors}

Transfection was conducted at three concentrations $(60,100$ and $150 \mathrm{pmol})$ and two time-points (12 and $24 \mathrm{~h}$ ). As expected, rno-miR-210-3p expression in the $24 \mathrm{~h} 210 \mathrm{M}$ (transfection with rno-miR-210-3p mimic) 100p group was significantly higher $(P<0.01)$, compared to that in the other groups (Fig. 2a). Conversely, expression of rno-miR-210-3p in the $24 \mathrm{~h}$ 210I (transfection with rno-miR-210-3p inhibitor) 150p group was significantly lower than that in the other groups $(P<0.01, P<0.05$; Fig. 2b). Green fluorescence was observed in the $24 \mathrm{~h} 210 \mathrm{M} 100 \mathrm{p}$ and $24 \mathrm{~h}$ 210I 150p groups (Fig. 2c, d). Accordingly, these two conditions were used for subsequent transfection experiments.

\section{Regulation of genes by rno-miR-210-3p in BRL cells}

After transfection of $100 \mathrm{pmol} /$ dish mimic or $150 \mathrm{pmol} / \mathrm{dish}$ inhibitor into rat hepatocyte lines for $24 \mathrm{~h}$, ISCU, Rap1b, ATP1b1, GPD1, E2F3, RAD52, PSMB6 and GPD2 levels appeared downregulated (Fig. 3a-h; $P<0.05$ or $P<0.01$ ). Upon inhibition of rno-miR-210-3p, ISCU, GPD1, E2F3, RAD52, PSMB6 and GPD2 levels were increased (Fig. $3 \mathrm{~b}-\mathrm{f} ; P<0.05$ or $P<0.01$ ) 


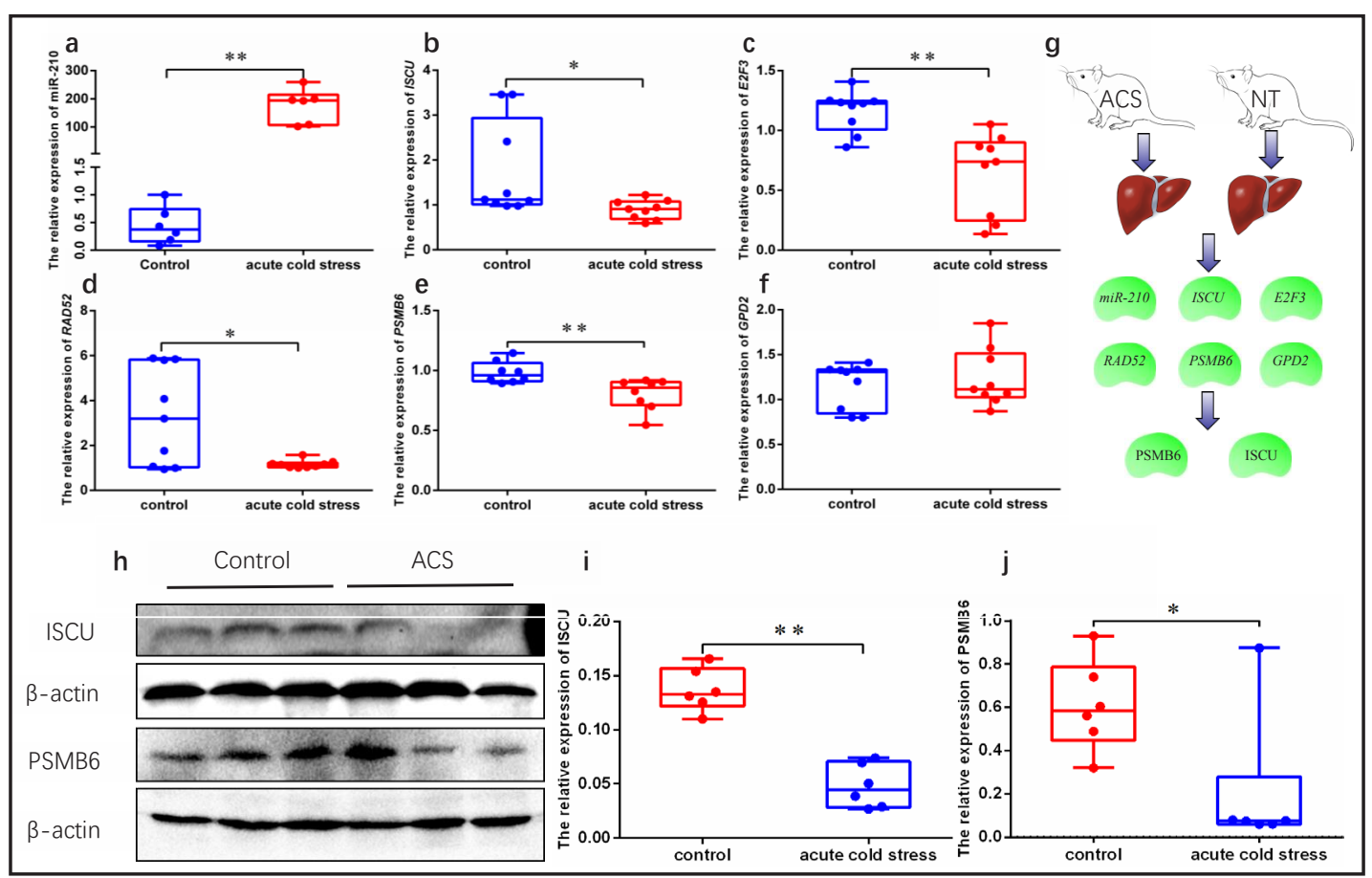

Fig. 4. Relative protein expression of PSMB6 and ISCU in rat liver and relative mRNA/miRNA expression of rno-miR-210-3p, ISCU, E2F3, RAD52, PSMB6 and GPD2. (a) miRNA expression of rno-miR-210-3p was detected via qRT-PCR in rat liver (control, $n=6, A C S, n=6$ ) and normalized to cel-miR-39-5p. (b-f) Relative expression levels of ISCU, E2F3, RAD52, PSMB6 and GPD2 were detected using qRT-PCR in rat liver (control, $\mathrm{n}=6, \mathrm{ACS}, \mathrm{n}=6$ ) and normalized to GAPDH. (g) Carrier experimental flow. (h) The control group was exposed to normal temperature (NT). The ACS group was subjected to acute cold stress $\left(4^{\circ} \mathrm{C}, 12 \mathrm{~h}\right)$. (i-j) Relative expression of ISCU and PSMB6. Protein expression levels were detected using Western blot analysis in rats' liver under ACS (Acute cold stress) and NT (Normal temperature). Relative protein expression was measured using ImageJ software (http://imagej.nih.gov/ij/), and was normalized to $\beta$-actin. Data presented as mean \pm SEM. $(n=6)$. All data throughout this Fig. are analyzed using T test. ${ }^{*} P<0.05,{ }^{* *} P<0.01$.

and Rap1b and GPD1 were downregulated (Fig. 3a and $\mathrm{g}, P<0.05$ ) while ATP1b1 showed no significant alterations in expression (Fig. $3 \mathrm{~h}$ ). ISCU and PSMB6 proteins showed a consistent trend with the corresponding gene expression patterns (Fig. 3i-k; $P<0.05, P<0.01$ ). Our findings suggest that rno-miR-210-3p affects specific activities by regulating changes in the associated genes.

Effects of ACS on rno-miR-210-3p and target gene levels

Relative expression of rno-miR-210-3p was significantly increased in ACS conditions $(P<0.01$, Fig. 4a). Under ACS, relative expression of ISCU, RAD52, E2F3 and PSMB6 showed a significant decrease $(P<0.01, P<0.05$, Fig. $4 \mathrm{~b}-\mathrm{e})$ while that of $G P D 2$ was not markedly different between the control and ACS groups (Fig. 4f). Consistent with gene expression patterns, we observed a significant decrease in the relative protein expression of ISCU and PSMB6 $(P<0.01, P<0.05$, Fig. 6g, h). These results were in agreement with data obtained with BRL cells.

Effects of rno-miR-210-3p on glycolysis and mitochondrial metabolism

Following treatment of BRL cells with mimic (100 pmoL/dish) or inhibitor (150 pmoL/dish), the glycolysis process was significantly promoted (Fig. 5a). The corresponding acidification rate (ECAR) was determined byaddingglucose, amphotericin and 2-deoxyglucose (2-DG) to reflect changes in the glycolytic process. Basal glycolysis rates of BRL cells in the $210 \mathrm{M}$ and 210I groups were slightly higher than those in the NC (transfection with MNC) 
Fig. 5. Measurement of glucose flux in mitochondrial and glycolysis using the XFe24 Extracellular Flux Analyzer in rat hepatocyte lines. NC, transfection with Mimic (duolex) Negative Control (a) Rat hepatocyte cell lines were treated with mimic or inhibitor for $24 \mathrm{~h}$. A representative curve is shown (n $=4$ replicates for each cell culture condition). Four independent experiments were performed. (b) Differences between initial ECAR (Rate 3) and ECAR (Rate 11) after 2-DG treatment represent the basal glycolysis rate of BRL cells. (c) Differences between ECAR (Rate 5) after exogenous glucose treatment and ECAR (Rate 11) after 2-DG treatment represent the glucose metabolism rate of

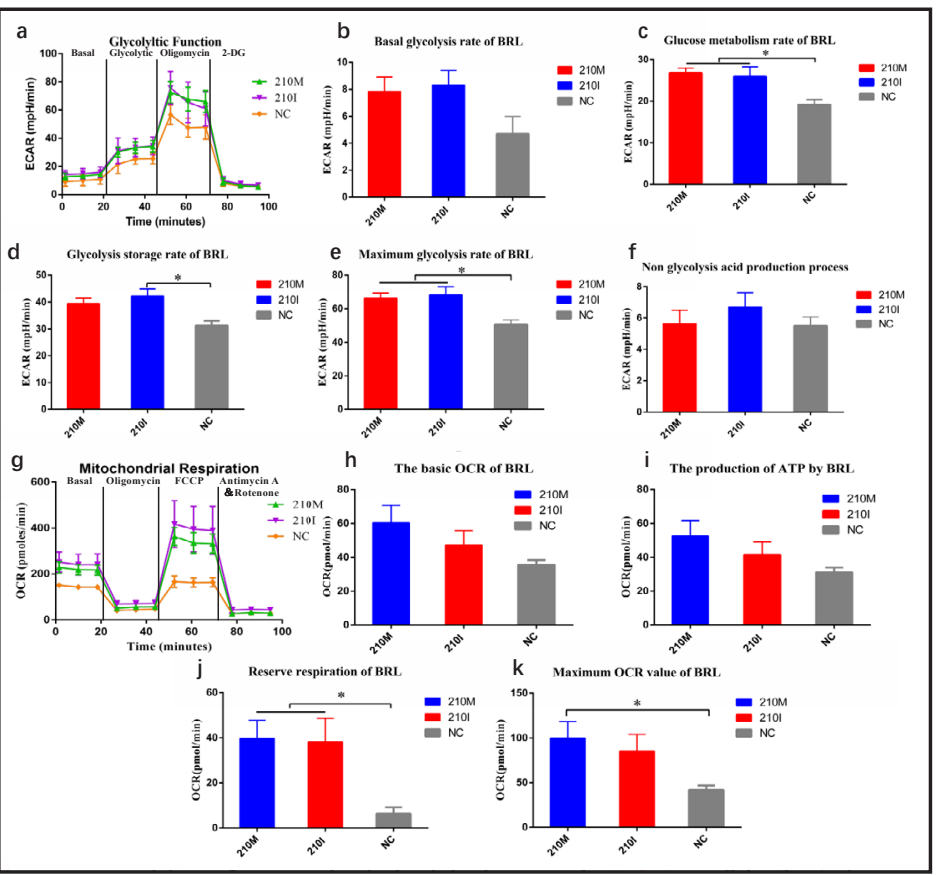
BRL cells. (d) Differences between ECAR (Rate 7) after oligomycin treatment and ECAR (Rate 5) after 2-DG treatment represent the glycolysis storage rate of BRL cells. (e) Differences between ECAR (Rate 7) after oligomycin treatment and ECAR (Rate 11) after 2-DG treatment represent the maximum glycolysis rate of BRL cells. (f). ECAR (Rate 12) following 2-DG treatment represents the non-glycolysis acid production rate of BRL cells. (g) Rat hepatocyte lines were treated with mimic or inhibitor for $24 \mathrm{~h}$. A representative curve is shown $(\mathrm{n}=4$ replicates for each cell culture condition) and four independent experiments were performed. (h) Difference between initial OCR (Rate 3) and OCR (Rate 11) after Rotenone\&Antimycin A treatment represents basic OCR of BRL cells. (i) Difference between initial OCR (Rate 3) and OCR (Rate 5) after oligomycin treatment represents ATP production by BRL cells. (j) Difference between OCR (Rate 8) after FCCP treatment and initial OCR (Rate 3) after Rotenone\&Antimycin A treatment represents reserve respiration of BRL cells. (k) Difference between OCR (Rate 8) after FCCP treatment and OCR (Rate 11) represents the maximum OCR value of BRL cells. All values are mean \pm SEM. in the bar graph $(\mathrm{n}=4)$. Data were analyzed using ANOVA $\left({ }^{* *} P<0.01 ;{ }^{*} P<0.05, \mathrm{n}=4\right)$.

group, but not to a significant extent (Fig. 5b). Glucose metabolism and maximum glycolysis rates of BRL cells between the $210 \mathrm{M}$ and $210 \mathrm{I}$ groups were not markedly different, but higher than those of the NC group $(P<0.05$, Fig. $5 c$ c e). The glycolysis storage rate of BRL cells was higher in the 210I group than in the NC group $(P<0.05)$. While the rates measured for $210 \mathrm{M}$ were relatively lower than the 210I group and higher than the NC group, the differences between the $210 \mathrm{M}$ group and the other two groups were not statistically significant (Fig. $5 \mathrm{~d})$. Moreover, differences in non-glycolytic acid production processes among the $210 \mathrm{M}$, $210 \mathrm{I}$ and NC groups were not statistically significant, but the measured values for the 210I group were higher than those in the NC group (Fig. 5f).

The overall OCR curve of BRL cells was significantly higher than that of the control group following mimic (100 pmoL/dish) or inhibitor (150 pmoL/dish) treatment. Our results showed that OCR of BRL cells treated with the oligo is significantly decreased, but there were no apparent differences among the three groups. After treatment with the uncoupling agent, FCCP, cellular OCR was markedly increased. OCR curves for the 210I and 210M groups were significantly higher than those for the NC group $(P<0.05)$. Moreover, cellular OCR was dramatically decreased after the addition of Rotenone and Antimycin A, but no significant differences were evident among the three groups (Fig. 5g). Basic OCR and production of ATP were comparable among the groups, although that of the $210 \mathrm{M}$ group was higher relative to the $210 \mathrm{I}$ and NC groups (Fig. $5 \mathrm{~h}, \mathrm{i}$ ). Reserve respiration rates of BRL cells in the $210 \mathrm{M}$ and 


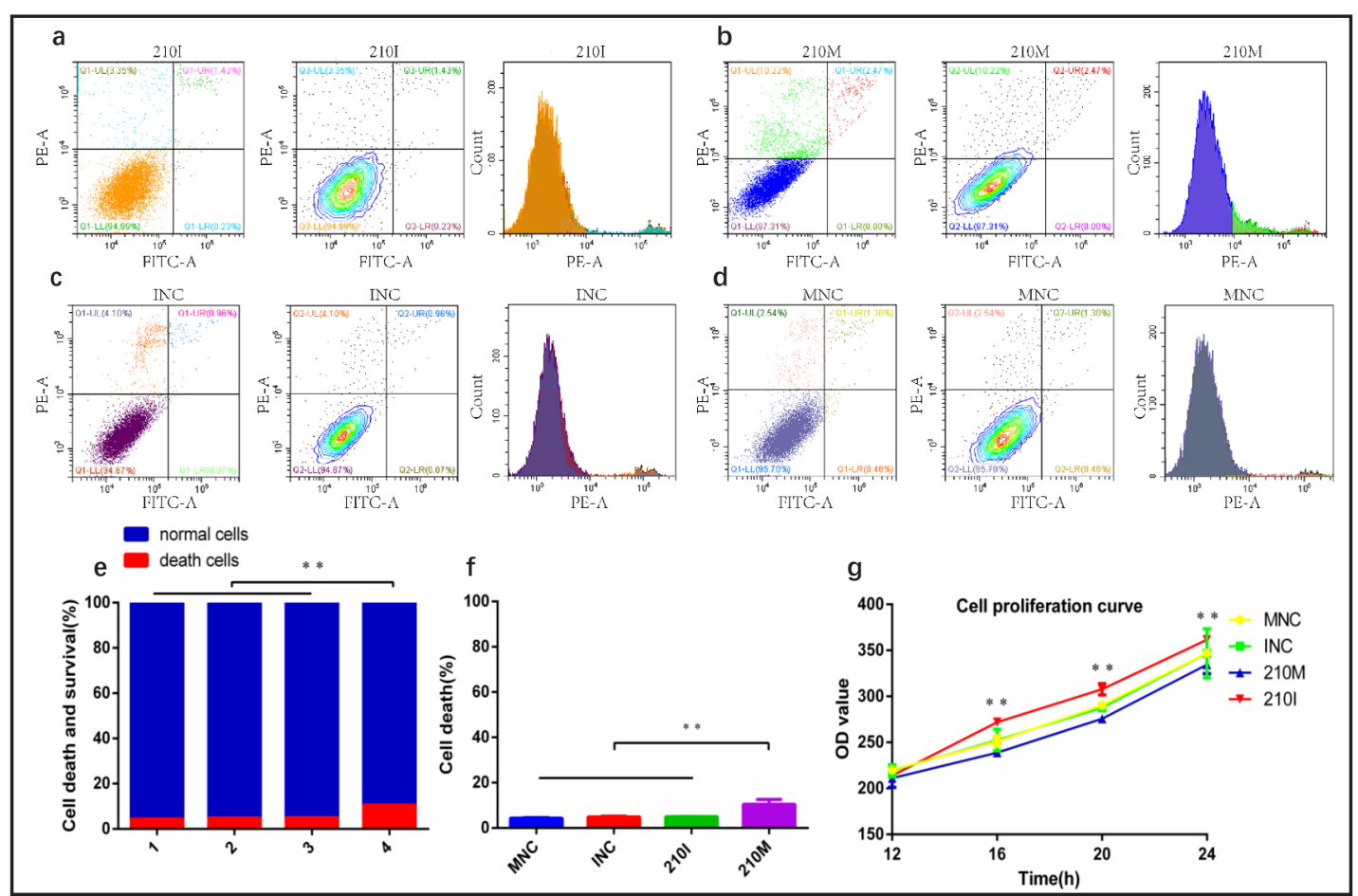

Fig. 6. rno-miR-210-3p overexpression and inhibition affect cell death. Cell death was measured via FACS after labeling BRL cells with FITC-annexin V (FITC-A, X-axis) and propidium iodide (PI, Y-axis). The effect of rno-miR-210-3p on BRL cells proliferation between 12 and $24 \mathrm{~h}$ was detected using WST-1 method. (a) Cell death following transfection with 210I (inhibitor) for $24 \mathrm{~h}$. (b) Cell death following transfection with 210M (mimic) for 24 h. (c) Cell death following transfection with INC (inhibitor control) for $24 \mathrm{~h}$. (d) Cell death following transfection with MNC (negative control mimic) for $24 \mathrm{~h}$. (e) Proportions of normal and dead cells.1, MNC; 2, INC; 3, 210I; 4, 210M. ${ }^{* *} \mathrm{P}<0.01$ vs 1,2,3. (f) Percentage of apoptotic cells in the indicated culture conditions. (g) Proliferation of BRL cells from 12 to 24 h. ${ }^{* *} P<0.01210 \mathrm{I}$ vs $210 \mathrm{M}$. No significant differences between INC and MNC groups. All values are mean \pm SEM. in the bar graph $(n=3)$. Data was analyzed using ANOVA. In cases where overall F-tests were significant $(P<0.05)$, post hoc comparisons using Tukey's method of adjustment were conducted to determine the location of significant pairwise differences $(* * P<0.01)$.

210I groups were not markedly different, but were higher than those in NC group $(P<0.05$, Fig. 5j). Maximum OCR was significantly higher in the $210 \mathrm{M}$ groups than in the NC group $(P<0.05$, Fig. $5 \mathrm{k})$.

\section{Effects of rno-miR-210-3p on apoptosis and proliferation}

In the 210I, MNC and INC treatment groups, cell populations showed no tendency to move (Fig. 6a, c and d) while cell populations of the 210M group tended to move up two quadrants (Fig. 6b). Cell death in the $210 \mathrm{M}$ group was significantly higher than that in the other three groups (Fig. 6e and f). The rno-miR-210-3p inhibitor promoted cell proliferation whereas rno-miR-210-3p mimic inhibited proliferation to a significant extent, especially at 16, 20 and 24 h (Fig. 6g).

\section{Discussion}

In the current study, 12 -h periods at $4^{\circ} \mathrm{C}$ were used to simulate ACS for the study of cold stress-associated mechanisms. Conditions of ACS may lead to hypoxia, metabolic disorder and apoptosis in cells [21]. qRT-PCR was employed to detect rno-miR-210-3p in liver 
under both ACS and normal conditions. The relative expression of rno-mir-210$3 p$ under ACS was significantly higher than that of the control group subjected to normal temperature conditions $(P<0.01)$. Targetscan, miRanda, miRDB and PicTar programs were applied to predict the target genes of rno-miR-210-3p, focusing on cell pro-

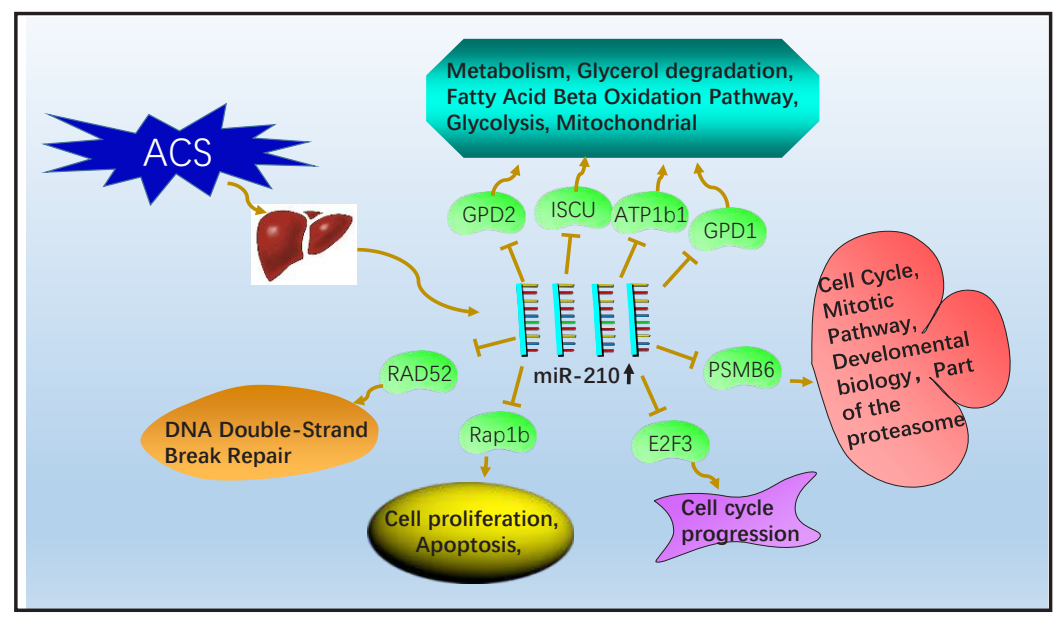

Fig. 7. Schematic of the pathways of rno-miR-210-3p under acute cold stress liferation, apoptosis, immunity and energy metabolism processes, which are closely associated with ACS.

To simulate the rapid increase in rno-miR-210-3p under ACS conditions, rno-miR-210$3 p$ was upregulated in BRL cells via transfection. In response, relative levels of $I S C U, R a p 1 b$, $A T P 1 b 1, G P D 1, E 2 F 3, R A D 52, P S M B 6$ and GPD2 were significantly reduced $(P<0.05, P<0.01)$, clearly indicating that rno-miR-210-3p inhibits the expression of specific genes under ACS to regulate cellular activities in humans and animals (Fig. 7). Changes in a single gene may not be particularly effective in the regulatory response to ACS. However, rno-miR-210-3p can simultaneously regulate various genes under adverse conditions to allow the body to rapidly adapt to changes in ambient temperature. The rno-miR-210-3p inhibition test facilitated the identification of five potential target genes (ISCU, E2F3, RAD52, PSMB6 and GPD2) related to energy metabolism [15] and involved in electron transport (respiratory) chain [22]. E2F3 belongs to the E2F family and plays critical roles as a cell cycle regulator and tumor suppressor [23]. RAD52 is important for invasion of the DNA chain during homologous recombination. RAD52 aids in repairing the gap between DNA double- and single-strands to help with RAD51 positioning, thus initiating homologous strand exchange [24]. PSMB6, which constitutes part of the $20 \mathrm{~S}$ proteasome core area, contributes to the complete assembly of the complex [25]. The protein (encoded by GPD2 and GPD1) constitutes a glycerol phosphate shuttle and can re-oxidize NADH during the glycolysis process [26, 27]. In earlier experiments, when rats were subjected to ACS, the liver, which is the most important heat-producing organ of the body, enhanced energy metabolism activity and heat production [28, 29]. Expression of miR-210 was rapidly increased in order to control levels of GPD1, GPD2 and ISCU. This led to decreased activity of the body's respiratory chain [30] and slowed energy metabolism to prevent oxidative damage of liver caused by excessive mobilization. However, upregulation of miR-210 is known to suppress expression of RAD52, resulting in DNA single-strand or double-strand breaks that are difficult to repair and cause DNA damage in the liver. The decrease in PSMB6 suggests that conditions of ACS may cause a reduction in proteasome synthesis, thereby reducing degradation of key proteins in signaling pathways to maintain metabolic activity in cells [31]. Simultaneously, the proteasome is involved in the formation of the active form of NF- $\kappa B$ [32]. Thus, ACS may reduce inflammation to further enhance the body's ability to adapt to extreme conditions. Rno-miR-210-3p may also regulate the cell cycle by inhibiting expression of E2F3 [33].

No significant differences were evident between the $210 \mathrm{M}$ and 210I groups in terms of basal glycolysis rate, glucose metabolism, glycolysis storage and maximum glycolysis of BRL cells. However, all values were higher than those of the NC group and those of the 210I group were higher than the $210 \mathrm{M}$ group, which may be related to inhibition of GPD1 and GPD2 in the 210M group to reduce NADH formation [26]. Non glycolytic acid production 
in the 210I groups was higher than that in the $210 \mathrm{M}$ and NC groups, indicating that when the body is subjected to ACS, the dramatic increase in rno-mir-210-3p contributes to glycolysis in the body and controls the acid production of cells. However, high non-glycolytic acid production is not conducive to producing long-lasting energy. During mitochondrial respiratory monitoring, basic OCR and production of ATP in the $210 \mathrm{M}$ and 210I groups were higher than those of the NC group, with the highest values obtained for the 210M group. With increased expression of rno-miR-210-3p, oxygen consumption and ATP production of BRL cells increased, enhancing resistance to ACS in the body [34]. Reserve respiration in the $210 \mathrm{M}$ and 210I groups and maximum OCR in the 210M group were significantly higher than that in the NC group. Our results suggest that when rats are subjected to ACS, upregulation of rno-miR-210-3p promotes glycolysis and mitochondrial respiration in liver. Rno-miR-210$3 p$ also enhances the thermogenic capacity and controls acid production of liver to improve the capacity of resistance to ACS in the body [35].

Earlier proliferation and apoptosis tests in BRL cells have revealed that cell proliferation is increased slowly and parts of cells undergo death after upregulation of rno-miR-210-3p [36]. This finding may be attributed to reduction of E2F3 and proteasome activity, which affects the cell cycle [37]. DNA chain damage is increased upon suppression of RAD52. The cell proliferation rate is significantly accelerated upon downregulation of rno-miR-210-3p, possibly due to an increase in levels of ISCU and GPD2, which are associated with energy generation [38]. Metabolic activity is enhanced, and the rates of division and proliferation accelerated with declining expression of rno-miR-210-3p.

\section{Conclusion}

Through a series of experiments, we showed that under acute cold conditions, rnomiR-210-3p is upregulated, in turn, modulating the expression of ISCU, Rap1b, ATP1b1, GPD1, E2F3, RAD52, PSMB6 and GPD2, promoting glycolysis of liver and enhancing the mitochondrial respiratory capacity of cells. However, upregulation of rno-miR-210-3p may additionally cause cell death. Our results collectively suggest that increased rno-miR-210-3p expression and consequent regulation of specific target genes is a favored mechanism used by the body to cope with ACS.

\section{Acknowledgements}

We are grateful to Harbin Medical University for the XFe24 Extracellular Flux Analyzer. This work was supported by the National Natural Science Foundation of China (grant Nos. 31372398, 31471586 and 31672513) and the National Key R\&D Program (grant No. 2016YFD0501210).

\section{Disclosure Statement}

No conflict of interests exists.

\section{References}

-1 Serrat MA, Williams RM, Farnum CE: Exercise mitigates the stunting effect of cold temperature on limb elongation in mice by increasing solute delivery to the growth plate. J Appl Physiol (1985) 2010;109:18691879.

-2 Bansal S, St Clair JB, Harrington CA, Gould PJ: Impact of climate change on cold hardiness of Douglas-fir (Pseudotsuga menziesii): environmental and genetic considerations. Glob Chang Biol 2015;21:3814-3826. 
3 Brazaitis M, Eimantas N, Daniuseviciute L, Mickeviciene D, Steponaviciute R, Skurvydas A: Two strategies for response to 14 degrees $\mathrm{C}$ cold-water immersion: is there a difference in the response of motor, cognitive, immune and stress markers? PLoS One 2014;9:e109020.

4 Garcia-Diaz EC, Gomez-Quiroz LE, Arenas-Rios E, Aragon-Martinez A, Ibarra-Arias JA, del Socorro IR-MM: Oxidative status in testis and epididymal sperm parameters after acute and chronic stress by cold-water immersion in the adult rat. Syst Biol Reprod Med 2015;61:150-160.

-5 Wang J, Chen Y, Zhang W, Zheng G, Meng S, Che H, Ke T, Yang J, Chen J, Luo W: Akt activation protects liver cells from apoptosis in rats during acute cold exposure. Int J Biol Sci 2013;9:509-517.

6 Sesti-Costa R, Ignacchiti MD, Chedraoui-Silva S, Marchi LF, Mantovani B: Chronic cold stress in mice induces a regulatory phenotype in macrophages: correlation with increased 11beta-hydroxysteroid dehydrogenase expression. Brain Behav Immun 2012;26:50-60.

7 Leung AK, Sharp PA: microRNAs: a safeguard against turmoil? Cell 2007;130:581-585.

-8 Leung AK, Sharp PA: MicroRNA functions in stress responses. Mol Cell 2010;40:205-215.

9 Dalmay T: Mechanism of miRNA-mediated repression of mRNA translation. Essays Biochem 2013;54:2938.

10 Friedman RC, Farh KK, Burge CB, Bartel DP: Most mammalian mRNAs are conserved targets of microRNAs. Genome Res 2009;19:92-105.

11 Emanueli C, Shearn AI, Laftah A, Fiorentino F, Reeves BC, Beltrami C, Mumford A, Clayton A, Gurney M, Shantikumar S, Angelini GD: Coronary Artery-Bypass-Graft Surgery Increases the Plasma Concentration of Exosomes Carrying a Cargo of Cardiac MicroRNAs: An Example of Exosome Trafficking Out of the Human Heart with Potential for Cardiac Biomarker Discovery. PLoS One 2016;11:e0154274.

-12 Tsang VH, Dwight T, Benn DE, Meyer-Rochow GY, Gill AJ, Sywak M, Sidhu S, Veivers D, Sue CM, Robinson BG, Clifton-Bligh RJ, Parker NR: Overexpression of miR-210 is associated with SDH-related pheochromocytomas, paragangliomas, and gastrointestinal stromal tumours. Endocr Relat Cancer 2014;21:415-426.

13 Dang K, Myers KA: The role of hypoxia-induced miR-210 in cancer progression. Int J Mol Sci 2015;16:63536372.

14 Luo R, Wang Y, Xu P, Cao G, Zhao Y, Shao X, Li YX, Chang C, Peng C, Wang YL: Hypoxia-inducible miR210 contributes to preeclampsia via targeting thrombospondin type I domain containing 7A. Sci Rep 2016;6:19588.

15 He M, Lu Y, Xu S, Mao L, Zhang L, Duan W, Liu C, Pi H, Zhang Y, Zhong M, Yu Z, Zhou Z: MiRNA-210 modulates a nickel-induced cellular energy metabolism shift by repressing the iron-sulfur cluster assembly proteins ISCU1/2 in Neuro-2a cells. Cell Death Dis 2014;5:e1090.

16 Wang H, Flach H, Onizawa M, Wei L, McManus MT, Weiss A: Negative regulation of Hif1a expression and TH17 differentiation by the hypoxia-regulated microRNA miR-210. Nat Immunol 2014;15:393-401.

17 Shang C, Hong Y, Guo Y, Liu YH, Xue YX: MiR-210 up-regulation inhibits proliferation and induces apoptosis in glioma cells by targeting SIN3A. Med Sci Monit 2014;20:2571-2577.

18 Wang J, Xu L, Yun X, Yang K, Liao D, Tian L, Jiang H, Lu W: Proteomic analysis reveals that proteasome subunit beta 6 is involved in hypoxia-induced pulmonary vascular remodeling in rats. PLoS One 2013;8:e67942.

19 Ullmann P, Qureshi-Baig K, Rodriguez F, Ginolhac A, Nonnenmacher Y, Ternes D, Weiler J, Gabler K, Bahlawane C, Hiller K, Haan S, Letellier E: Hypoxia-responsive miR-210 promotes self-renewal capacity of colon tumor-initiating cells by repressing ISCU and by inducing lactate production. Oncotarget 2016;7:65454-65470.

20 Zhen L, Guo W, Peng M, Liu Y, Zang S, Ji H, Li S, Yang H: Identification of cold-responsive miRNAs in rats by deep sequencing. J Therm Biol 2017;66:114-124.

-21 Boardman L, Sorensen JG, Terblanche JS: Physiological and molecular mechanisms associated with cross tolerance between hypoxia and low temperature in Thaumatotibia leucotreta. J Insect Physiol 2015;82:7584.

22 Yan R, Adinolfi S, Pastore A: Ferredoxin, in conjunction with NADPH and ferredoxin-NADP reductase, transfers electrons to the IscS/IscU complex to promote iron-sulfur cluster assembly. Biochim Biophys Acta 2015;1854:1113-1117.

-23 Lee M, Oprea-Ilies G, Saavedra HI: Silencing of E2F3 suppresses tumor growth of Her2+ breast cancer cells by restricting mitosis. Oncotarget 2015;6:37316-37334. 


\section{Cellular Physiology Cell Physiol Biochem 2018;46:2090-2102 \begin{tabular}{l|l} 
DOI: 10.1159/000489449 & $\begin{array}{l}\text { O } 2018 \text { The Author(s). Published by S. Karger AG, Basel } \\
\text { www.karger.com/cpb }\end{array}$
\end{tabular} \\ Guo et al.: The Favored Mechanism for Coping With Acute Cold Stress: Upregulation of MiR-210 in Rats}

24 Sugiyama T, Kantake N, Wu Y, Kowalczykowski SC: Rad52-mediated DNA annealing after Rad51-mediated DNA strand exchange promotes second ssDNA capture. EMBO J 2006;25:5539-5548.

25 Coux 0, Tanaka K, Goldberg AL: Structure and functions of the 20S and 26S proteasomes. Annu Rev Biochem 1996;65:801-847.

-26 Kota V, Rai P, Weitzel JM, Middendorff R, Bhande SS, Shivaji S: Role of glycerol-3-phosphate dehydrogenase 2 in mouse sperm capacitation. Mol Reprod Dev 2010;77:773-783.

27 Kota V, Dhople VM, Shivaji S: Tyrosine phosphoproteome of hamster spermatozoa: role of glycerol-3phosphate dehydrogenase 2 in sperm capacitation. Proteomics 2009;9:1809-1826.

-28 Churchill TA, Cheetham KM, Simpkin S, Green CJ, Wang LC, Fuller BJ: Liver metabolism in cold hypoxia: a comparison of energy metabolism and glycolysis in cold-sensitive and cold-resistant mammals. J Comp Physiol B 1994;164:396-404.

29 Ferrigno A, Di Pasqua LG, Bianchi A, Richelmi P, Vairetti M: Metabolic shift in liver: correlation between perfusion temperature and hypoxia inducible factor-1alpha. World J Gastroenterol 2015;21:1108-1116.

-30 Chan SY, Zhang YY, Hemann C, Mahoney CE, Zweier JL, Loscalzo J: MicroRNA-210 controls mitochondrial metabolism during hypoxia by repressing the iron-sulfur cluster assembly proteins ISCU1/2. Cell Metab 2009;10:273-284.

31 Schmidt M, Finley D: Regulation of proteasome activity in health and disease. Biochim Biophys Acta 2014;1843:13-25.

-32 Wu M, Bian Q, Liu Y, Fernandes AF, Taylor A, Pereira P, Shang F: Sustained oxidative stress inhibits NFkappaB activation partially via inactivating the proteasome. Free Radic Biol Med 2009;46:62-69.

-33 Ping Z, Lim R, Bashir T, Pagano M, Guardavaccaro D: APC/C (Cdh1) controls the proteasome-mediated degradation of E2F3 during cell cycle exit. Cell Cycle 2012;11:1999-2005.

-34 Morrison BA, Shain DH: An AMP nucleosidase gene knockout in Escherichia coli elevates intracellular ATP levels and increases cold tolerance. Biol Lett 2008;4:53-56.

35 Rui L: Energy metabolism in the liver. Compr Physiol 2014;4:177-197.

-36 Li Y, Yang C, Zhang L, Yang P: MicroRNA-210 induces endothelial cell apoptosis by directly targeting PDK1 in the setting of atherosclerosis. Cell Mol Biol Lett 2017;22:3.

37 Guo X, Wang X, Wang Z, Banerjee S, Yang J, Huang L, Dixon JE: Site-specific proteasome phosphorylation controls cell proliferation and tumorigenesis. Nat Cell Biol 2016;18:202-212.

38 Bothe JR, Tonelli M, Ali IK, Dai Z, Frederick RO, Westler WM, Markley JL: The Complex Energy Landscape of the Protein IscU. Biophys J 2015;109:1019-1025. 